\title{
Fibroblast growth factor-2 induces the activation of Src through Fes, which regulates focal adhesion disassembly
}

Shigeru Kanda ${ }^{1,4,5}$, Yasuyoshi Miyata ${ }^{2}$, Hiroshi Kanetake ${ }^{2}$, and Thomas E. Smithgall ${ }^{3}$

${ }^{1}$ Department of Molecular Microbiology and Immunology, Division of Endothelial Cell Biology, and ${ }^{2}$ Department of Urology, Nagasaki University Graduate School of Biomedical Science, 1-7-1 Sakamoto, Nagasaki 852-8501, Japan, and ${ }^{3}$ Department of Molecular Genetics and Biochemistry, University of Pittsburgh School of Medicine, E1240 Biomedical Science Tower, Pittsburgh, PA 15261

${ }^{4}$ Corresponding author;

Shigeru Kanda, M.D., Ph.D.,

Department of Molecular Microbiology and Immunology,

Division of Endothelial Cell Biology,

Nagasaki University Graduate School of Biomedical Science,

1-7-1 Sakamoto,

Nagasaki 852-8501,

Japan

Phone: +81958497340

Fax: +81958497343

E-mail: skanda-jua@umin.net

${ }^{5}$ Present address:

National Hospital Organization, Nagasaki Hospital

41-6 Sakuragi,

Nagasaki 850-8523

Japan

Phone: +81 (0)95 823-2261 
Fax: +81 (0)95 828-2616

E-mail: skanda-jua@umin.net

Abbreviations: FGF, fibroblast growth factor; FA, focal adhesion; FAK, focal adhesion kinase; siRNA, small interfering RNA; CCD, coiled-coil domain; YFP, yellow-fluorescent protein; SDS-PAGE, SDS-polyacrylamide gel electrophoresis. 


\section{Abstract}

Cell migration is regulated by focal adhesion (FA) turnover. Fibroblast growth factor-2 (FGF-2) induces FA disassembly in the murine brain capillary endothelial cell line, IBE, leading to FGF-2-directed chemotaxis. We previously showed that activation of Src and Fes by FGF-2 was involved in chemotaxis of IBE cells. In this study, we examined the interplay between Src and Fes. FGF-2 treatment decreased the number of FA in IBE cells, but not in cells expressing dominant-negative Fes (denoted KE5-15 cells). FGF-2 induced the activation of Src and subsequent binding to and phosphorylation of Cas in IBE cells, but not in KE5-15 cells. Focal adhesion kinase (FAK) activation and tyrosine phosphorylation by Src was also delayed in KE5-15 cells compared to parental cells. FGF-2 induced activation of Src within FA in IBE cells, but not in KE5-15 cells. Down-regulation of Fes or FAK using small interfering RNA diminished Src activation by FGF-2 within FA. These findings suggest that activation of Fes by FGF-2 enhances FAK-dependent activation of Src within FA, promoting FGF-2-induced disassembly of focal adhesions.

Keywords: FGF-2; Src; Fes; FAK; endothelial cell; focal adhesion, migration 


\section{Introduction}

Angiogenesis is a prerequisite for both normal development as well as pathophysiological processes, such as collateral formation following occlusion of arteries, diabetic retinopathy, and solid tumor growth [1-3]. A number of endothelial cell responses must be integrated for efficient angiogenesis, including proliferation, differentiation and migration [4]. Cell migration is coordinated by signaling events involving both growth factor receptors and integrins [5-7]. Many studies have emphasized the contribution of dynamic assembly and disassembly of focal adhesions to this response. Although multiple signaling molecules are incorporated within focal adhesions (FA), focal adhesion kinase (FAK) and Src play fundamental roles in focal adhesion turnover [8-10].

Fibroblast growth factors (FGFs) are important regulators of angiogenesis both in vitro and in vivo [11, 12]. To date, 23 distinct forms of FGF have been identified [13]. Prototypical FGFs, such as FGF-1 and FGF-2, are the most thoroughly studied FGFs in relation to angiogenesis. Four structurally-related receptor tyrosine kinases for FGFs have been reported, denoted FGF receptors (FGFR) 1 to 4 [14]. Of these, both FGFR-1 and 2 are expressed in endothelial cells, where they induce Src activation and chemotaxis [15-17]. Src has also been implicated in FGF-regulated migration and proliferation of fibroblasts [18-20]. We have previously shown that Fes, a non-receptor protein tyrosine kinase distinct from Src, also contributes to FGF-2-induced chemotaxis and differentiation of capillary endothelial cells [21]. In this study, we provide evidence that Fes activity is required for FGF-2-induced activation of Src and focal adhesion disassembly, providing the first mechanistic link between these protein-tyrosine kinases in FGF-induced regulation of vascular endothelial cell behavior. 


\section{Materials and methods}

\section{Materials}

Anti-vinculin monoclonal antibody (clone hVIN-1), anti-FLAG monoclonal antibody (M2), and anti- $\beta$-actin monoclonal antibody (clone AC-74) were purchased from Sigma Chemical Company, St. Louis, MO. Anti-Src polyclonal antibodies (N-16 and SRC2), anti-Fes polyclonal antibody (N-19), anti-Cas polyclonal antibody (C-20), and non-targeting control small interfering RNA (siRNA) were purchased from Santa Cruz Biotechnologies, Santa Cruz, CA. Anti-pY ${ }^{418}$ Src, anti-pY ${ }^{529}$ Src, anti-pY ${ }^{397}$ FAK, and anti-pY ${ }^{861}$ FAK polyclonal antibodies were obtained from BioSource International, Inc., Camarillo, CA. Anti-phosphotyrosine monoclonal antibody (clone 4G10) and anti-FAK monoclonal antibody (clone 4.47 for immunoprecipitation) were purchased from Upstate Cell Signaling Solutions, Lake Placid, NY. Anti-FAK monoclonal antibody (clone 77 for immunostaining and immunoblotting) was obtained from BD Bioscience, San Jose, CA. HiPerFect transfection reagent, Alexa $\quad$ Fluor 555-labeled negative control siRNA (5'-UUCUUCGAACGUGUCACGU-3'), two mouse FAK siRNAs (5'-GGUCCAAUGACAAGGUAUA-3' and 5'-GAUUAUUAAAGGUCUUUCA3'), and two mouse $\quad$ Fes $\quad$ siRNAs (5CCUGAGAAAGACGUACAA-3' $^{\prime}$ and 5'-GGAUGGAGACAAGGCCAAA-3') were purchased from Qiagen K.K., Tokyo, Japan.

\section{Cells and culture}

Parental IBE cells [15] and IBE cell lines expressing kinase-inactive mutant Fes (denoted KE5-15 cells) were cultured as described elsewhere [21]. A stable IBE cell line 
expressing kinase-inactive Src (denoted KDSrc-2 cells), which exhibits a dominant negative effect on FGF-2-induced activation of Src, was cultured as described [17].

\section{Transfection of siRNA}

IBE cells were transfected with siRNA using the HiPerFect reagent. To examine the transfection efficiency, Alexa Fluor 555-labeled control siRNA was tranfected into IBE cells and on the next day, cells were washed, fixed with paraformaldehyde, and examined for fluorescence. To downregulate Fes, IBE cells were grown in $10 \mathrm{~cm}$ dishes and transfected with control siRNA or mixture of two siRNA for Fes and cultured for two days. Cells were then suspended in serum-free medium and counted; $80 \%$ of the cells were used for assessment of Fes expression by immunoprecipitation followed by immunoblotting while the remaining $20 \%$ were used for indirect immunofluorescent staining for vinculin and activated Src. To downregulate FAK, IBE cells were transfected with a mixture of two FAK siRNAs or control siRNAs in 6-well plates. Two days later, cells were used either for indirect immunofluorescent staining for vinculin and activated Src or lysed for the assessment of FAK and $\beta$-actin expression by immunoblotting.

\section{Immunoprecipitation and immunoblotting}

Immunoprecipitation and immunoblotting were performed as described before [22]. In brief, cells suspended in serum-free medium were seeded onto fibronectin-coated dishes and cultured for various periods in the presence or absence of $20 \mathrm{ng} / \mathrm{ml}$ of FGF-2. Cells were lysed in Triton X-100 lysis buffer and kinase or focal adhesion proteins were immunoprecipitated and separated by SDS-polyacrylamide gel electrophoresis (SDS-PAGE). After transfer onto 
polyvinylidene difluoride membranes (Millipore, Bedford, MA), the blots were probed with antibodies as indicated in the Figures. Prior to reprobing, blots were stripped as described previously [21]. For the FAK blots, band intensities were determined from scanned images of the blots using NIH Image (version 1.64). The relative ratio of FAK tyrosine phosphorylation was calculated as the ratio of the phospho-FAK signals divided by the corresponding FAK protein signal; each value was then normalized to the ratio obtained from untreated, suspended cells.

\section{Indirect immunofluorescent staining}

Indirect immunofluorescent staining was performed as described previously [23]. Cells were seeded onto the surface of fibronectin-coated coverslips with or without FGF-2 (20 $\mathrm{ng} / \mathrm{ml}$ ) and cultured for indicated periods. Cells were washed with PBS, fixed with 3.7\% paraformaldehyde, and permeabilized with $0.1 \%$ Triton X-100. To visualize FA, cells were stained with an anti-vinculin monoclonal antibody. To examine FA turnover, vinculin-positive spots were counted in at least 200 cells for each condition. For co-localization experiments, cells were seeded onto fibronectin-coated coverslips and cultured for $2 \mathrm{~h}$ with or without FGF-2. Cells were fixed and stained with the anti-vinculin monoclonal antibody (mouse) and anti-Fes polyclonal antibody (goat) or anti-pY $\mathrm{Y}^{418}$ Src polyclonal antibody (rabbit). Mouse primary antibodies were visualized with corresponding secondary antibodies labeled with Alexa Fluor 488, while goat or rabbit primary antibodies were recognized with specific secondary antibody-Alexa Fluor 555 conjugates. 
The in vitro kinase assay for Src was described previously [16]. In brief, cells were seeded onto fibronectin-coated wells of 6-well plates with or without $20 \mathrm{ng} / \mathrm{ml} \mathrm{FGF-2.} \mathrm{After} 2$ h, cells were lysed in NP40 lysis buffer supplemented with 0.1\% 2-mercaptoethanol. Src was immunoprecipitated and incubated in kinase buffer supplemented with acid-denatured enolase and $\left[\gamma^{3}{ }^{32} \mathrm{P}\right]$ ATP, followed by SDS-PAGE. Gels were washed, fixed, treated with $1 \mathrm{M} \mathrm{KOH}$ to remove phosphorylated serine residues, and imaged using a BAS 5000 Image Analyzer followed by exposure to X-ray film.

\section{Statistical analysis}

Data are presented as the mean \pm SD. Differences between two groups were examined by Mann-Whitney's U test. A $P$ value less than 0.05 denoted the presence of a statistically significant difference. 


\section{Results}

FGF-2 induces disassembly of FA in IBE cells

We have previously shown that Fes activity was involved in FGF-2-directed chemotaxis of IBE cells [21]. FA disassembly is critical for cell migration, and FGF-2-treatment of IBE cells decreases the number of FA [23]. To elucidate the role of Fes in FA turnover by IBE cells, we studied the number of FA in IBE cells expressing dominant-negative Fes following culture on a fibronectin-coated surface. As shown in Fig. 1, control IBE cells formed FA in response to plating on fibronectin, and the number of FA was decreased in the presence of FGF-2 both $1 \mathrm{~h}$ and $2 \mathrm{~h}$ later. In contrast, IBE cells expressing dominant-negative Fes (denoted KE5-15 cells) formed FA in greater numbers than parental IBE cells and FGF-2 treatment did not affect the number of FA. This result suggests that Fes is required for FGF-2-induced disassembly of FA in these cells. Unlike FA formation, FGF-2-induced stress fiber formation was not altered between the control and KE5-15 IBE cell lines (data not shown).

FGF-2 induces Src activation, which is dependent on Fes activity

To determine the possible interplay between Fes and FAK/Src signaling in FA turnover, we first examined the kinase activity of Src in FGF-2-treated parental IBE cells and KE5-15 cells, which express dominant-negative Fes. As shown in Fig. 2 A, Src activity was increased by FGF-2-treatment in control IBE cells but not in KE5-15 cells, suggesting that Fes kinase activity is required for FGF-2-induced Src activation. Consistent with this observation, both basal and FGF-2-induced Src autophosphorylation at Tyr 418 were blocked in KE5-15 
cells (Figure 2 B, lower panels). We also examined whether the presence of kinase-defective Fes affected interaction of endogenous Src with p130 Cas, an adaptor protein involved in FA turnover [24]. As shown in Figure 2 B, co-precipitation of Cas with Src was dramatically reduced in the KE5-15 cells, which is likely due to the block in Src activity resulting from the presence of dominant-negative Fes. The tyrosine phosphorylation of Cas in KE5-15 cells was slightly elevated by FGF-2-treatment. However, the amount of Src-bound Cas was extremely low in KE5-15 cells compared to the control cell line. Therefore, it seems likely that this elevation of Cas tyrosine phosphorylation was unable to compensate for the loss of Src-Cas signaling required for FA disassembly in the KE5-15 cells.

Ligation of integrins induces FAK autophosphorylation at Y397, which leads to its activation and creates a binding site for Src via its SH2 domain [8]. Binding to FAK may contribute to Src activation by disrupting the inhibitory interaction between its SH2 domain and tyrosine-phosphorylated tail [25]. Once bound and activated, Src phosphorylates FAK at Y861 [26]. To determine whether Fes affects the interplay of Src and FAK, we examined the kinetics of FAK activation and phosphorylation by Src in parental IBE cells and KE5-15 cells. As shown in Fig. 3, FAK was rapidly phosphorylated on Y397 following adhesion to fibronectin in control IBE cells, and phosphorylation of this site decreased $2 \mathrm{~h}$ after seeding. FGF-2 did not increase the phosphorylation of Y397 further and in fact appeared to hasten its dephosphorylation. By contrast, phosphorylation of Y397 was weak in KE5-15 cells at 30 min and was slightly increased at $2 \mathrm{~h}$, suggesting that Y397 phosphorylation is delayed by the presence of the Fes dominant-negative mutant. FGF-2 treatment did not affect the phosphorylation of FAK Y397 phosphorylation in KE5-15 cells. Adhesion-induced phosphorylation of FAK Y861 was also readily observed after 30 min in parental IBE cells, 
although this site remained phosphorylated at $2 \mathrm{~h}$ and was prolonged in response to FGF-2 treatment, suggesting that FGF-2-activated Src may be involved. Although adhesion-dependent phosphorylation of FAK Y861 occurred with similar kinetics in KE5-15 cells, the FGF-2-induced increase in phosphorylation at $2 \mathrm{~h}$ was not observed. Together, these data implicate Fes in the adhesion-dependent activation of FAK through Src.

FGF-2 activates Src within FA, which is dependent on Fes and FAK

To elucidate the role of Fes and FAK in FGF-2-induced activation of Src, we employed siRNA technology. Using Alexa Fluor 555-labeled negative control siRNA, we first established conditions that allowed for a transfection efficiency of more than 90\% in IBE cells (data not shown). Next, we transfected IBE cells with either control siRNA or Fes siRNA. Two days later, cells were seeded onto fibronectin-coated culture plates and Fes expression as well as activation of Src at FA were examined. As shown in Fig. 4 A, the Fes siRNA efficiently downregulated Fes protein expression, while the contol siRNA was without effect. We then examined the localization of Fes in IBE cells. In cells transfected with control siRNA, Fes localized to the cytoplasm as well as FA (Fig. 4 B). We also determined the specificity of immunofluorescent staining with the Src phosphospecific antibody, pY418. Our previous study has shown that FGF-2 activated Src within FA, which was followed by the activation of mitogen-activated protein kinase within FA [17]. As shown in Fig. 4 C, focal adhesions in FGF-2-treated mock-transfected cells stained positively with the anti-pY418 Src antibody, indicating that active Src is present within FA. In contrast, the antibody failed to detect activated Src in FA of FGF-2-treated cells expressing dominant-negative Src (KDSrc-2 cells). Fig. 4D shows that Src activation in focal adhesions was also completely blocked in cells 
transfected with Fes siRNA, indicating that Fes is required for FGF-2-induced Src activation within FA. As a control, we also determined the effect of FAK knock-down on Src activation within FA. As shown in Fig. 5, suppression of FAK protein expression with siRNA also blocked FGF-2-induced Src activation within FA. 


\section{Discussion}

In this study, we provide new evidence that the c-Fes tyrosine kinase may regulate FA turnover in IBE capillary endothelial cells by modulating Src and FAK signaling. Several previous studies have established that oligomerization is essential for the kinase and biological activities of c-Fes. Importantly, kinase-dead Fes has been shown to form mixed oligomers with wild-type Fes and suppresses its autophosphorylation [27]. Directly relevant to this study is our prior observation that expression of kinase-inactive Fes abolished FGF-2-induced autophosphorylation of endogenous Fes in IBE cells, suggesting that kinase-inactive Fes oligomerized with endogenous Fes leading to the blockade of FGF-2-directed chemotaxis in this system [21]. Experiments with a dominant-negative mutant provide evidence that Fes activity is required for Src activation by FGF-2, which in turn regulates Src interaction with the FA protein p130 Cas (Fig. 2). Perhaps most striking was the observation that targeted knock-down of Fes protein expression with siRNA almost completely blocked FGF-2-regulated Src activation within FA (Fig. 4). The magnitude of this effect was virtually identical to that observed when FAK itself was suppressed with siRNA (Fig. 5).

In parental IBE cells, FAK activation (autophosphorylation of Y397) was observed 30 minutes after plating on fibronectin and was independent of FGF-2-treatment. This initial rapid activation was followed by dephosphorylation of this tyrosine residue at $2 \mathrm{~h}$. In addition, we observed continuous, strong phosphorylation of Y861, a known phosphorylation site for c-Src on FAK (Fig. 3). These kinetics are consistent with the finding that FAK activation leads to the recruitment and activation of Src by disruption of the intramolecular inhibitory interaction between its SH2 domain and C-terminal tail [25]. In contrast, maximal 
phosphorylation of Y397 occurred much later in the presence of dominant-negative Fes, and overall Y861 phosphorylation was reduced $2 \mathrm{~h}$ after fibronectin plating in the presence and absence of FGF-2. From these results, it is clear that autophosphorylation of FAK was largely regulated by cell adhesion to fibronectin, and was also affected by Fes kinase activity. However, Src activation and subsequent phosphorylation of FAK at $2 \mathrm{~h}$ after plating was dependent on FGF-2-treatment in IBE cells, but not in KE5-15 cells. Together, these observations suggest a model in which FGF-2-induced Fes activation contributes to Src activation through FAK in FA, promoting their disassembly (Fig. 6). Indeed, dominant-negative Fes prevents the FA disassembly normally observed following treatment of IBE cells with FGF-2 (Fig. 1).

While the direct biochemical target for Fes in FA is not clear, FAK appears to be a strong candidate based on the effect of dominant-negative Fes on the kinetics of FAK activation in IBE cells. One conclusion is that dominant-negative Fes may prevent phosphorylation of FAK by endogenous Fes in response to integrin engagement, thus delaying FAK activation. Interestingly, downregulation of endogenous Fes protein levels by siRNA in IBE cells did not decrease the level of FAK Y397 phosphorylation, supporting a requirement for the presence of the Fes protein in the inhibitory mechanism (data not shown). However, downregulation of Fes by siRNA did suppress FA formation. Taken together, these observations suggest that the presence of the Fes protein provides a necessary scaffolding function to recruit additional signaling partners required for FA turnover in response to FAK activation. This hypothesis is currently under investigation.

In summary, the present study emphasizes the significance of Fes in an important angiogenic response of endothelial cells, namely migration in response to FA disassembly. Fes also acts as a mediator of angiogenic stimuli promoted by angiopoietins, sonic hedgehog, and 
stromal cell-derived factor-1 $\alpha$ [22, 28-30]. Fes may represent a previously unrecognized endothelial cell-specific molecular target for anti-angiogenic therapy. Antagonists of Fes kinase activity may block signals through these and other proangiogenic pathways. 


\section{Acknowledgements}

We are grateful to T. Shimogama, M. Yoshimoto, and members of Nagasaki University Radioisotope Center for their skilled outstanding help. This work was supported by Grants-in-Aid for Scientific Research from the Japan Society for the Promotion of Science (to S.K.) and by National Institutes of Health grant CA101828 (to T.E.S). 


\section{References}

[1] P. Carmeliet, Angiogenesis in health and disease, Nat Med. 9 (2003) 653-660.

[2] R.K. Jain, Molecular regulation of vessel maturation, Nat Med. 9 (2003) 685-693.

[3] J. Folkman, Angiogenesis-dependent diseases, Semin Oncol. 28 (2001) 536-542.

[4] L. Beck Jr, P.A. D’Amore, Vascular development: cellular and molecular regulation,

FASEB J. 11 (1997) 365-373.

[5] K.M. Yamada, S. Miyamoto, Integrin transmembrane signaling and cytoskeletal control,

Curr Opin Cell Biol. 7 (1995) 681-689.

[6] F.G. Giancotti, E. Ruoslahti, Integrin signaling, Science 285 (1999) 1028-1032.

[7] D.J. Webb, J.T. Parsons, A.F. Horwitz, Adhesion assembly, disassembly and turnover in

migrating cells -- over and over and over again, Nature Cell Biol. 4 (2002) E97-E100.

[8] M.P. Playford, M.D. Schaller, The interplay between Src and integrins in normal and tumor biology, Oncogene 23 (2004) 7928-7946.

[9] M.C. Frame, Newest findings on the oldest oncogene; how activated src does it, J Cell Sci. 117 (2004) 989-998.

[10] S.K. Mitra, D.A. Hanson, D.D. Schlaepfer, Focal adhesion kinase: in command and control of cell motility, Nat Rev Mol Cell Biol. 6 (2005) 56-68. 
[11] S. Javerzat, P. Auguste, A. Bikfalvi, The role of fibroblast growth factors in vascular development, Trends Mol Med. 8 (2002) 483-489.

[12] M. Presta, P. Dell'Era, S. Mitola, E. Moroni, R. Ronca, M. Rusnati, Fibroblast growth factor/fibroblast growth factor receptor system in angiogenesis, Cytokine Growth Factor Rev. 16 (2005) 159-178.

[13] N. Itoh, D.M. Ornitz, Evolution of the Fgf and Fgfr gene families, Trends Genet. 20 (2004) 563-569.

[14] V.P. Eswarakumar, I. Lax, J. Schlessinger, Cellular signaling by fibroblast growth factor receptors, Cytokine Growth Factor Rev. 16 (2005) 139-149.

[15] S. Kanda, E. Landgren, M. Ljungstr öm, L. Claesson-Welsh, Fibroblast growth factor receptor 1-induced differentiation of endothelial cell line established from tsA58 large T transgenic mice, Cell Growth Differ, 7 (1996) 383-395.

[16] T. Nakamura, Y. Mochizuki, H. Kanetake, S. Kanda, Signals via FGF receptor 2 regulate migration of endothelial cells, Biochem Biophys Res Commun. 289 (2001) 801-806.

[17] T. Shono, H. Kanetake, S. Kanda, The role of mitogen-activated protein kinase activation within focal adhesions in chemotaxis toward FGF-2 by murine brain capillary endothelial cells, Exp Cell Res. 264 (2001) 275-283. 
[18] T.M. LaVallee, I.A. Prudovsky, G.A. McMahon, X. Hu, T. Maciag, Activation of the MAP kinase pathway by FGF-1 correlates with cell proliferation induction while activation of the Src pathway correlates with migration, J Cell Biol. 141 (1998) 1647-1658.

[19] L. Liu, C. Huang, X. Zhan, Src is required for cell migration and shape changes induced by fibroblast growth factor 1, Oncogene 18 (1999) 6700-6706.

[20] D.M. Kilkenny, J.V. Rocheleau, J. Price, M.B. Reich, G.G. Miller, c-Src regulation of fibroblast growth factor-induced proliferation in murine embryonic fibroblasts, J Biol Chem. 278 (2003) 17448-17454.

[21] S. Kanda, E.C. Lerner, S. Tsuda, T. Shono, H. Kanetake, T.E. Smithgall, The nonreceptor protein-tyrosine kinase c-Fes is involved in fibroblast growth factor-2-induced chemotaxis of murine brain capillary endothelial cells, J Biol Chem. 275 (2000) 10105-10111.

[22] S. Kanda, Y. Miyata, Y. Mochizuki, T. Matsuyama, H. Kanetake, Angiopoietin 1 is mitogenic for cultured endothelial cells, Cancer Res. 65 (2005) 6820-6827.

[23] S. Kanda, Y. Miyata, H. Kanetake, Role of focal adhesion formation in migration and morphogenesis of endothelial cells, Cell Signal. 16 (2004) 1273-1281.

[24] D. Chodniewicz, R.L. Klemke, Regulation of integrin-mediated cellular responses through assembly of a CAS/Crk scaffold, Biochim Biophys Acta. 1692 (2004) 63-76. 
[25] J.W. Thomas, B. Ellis, R.J. Boerner, W.B. Knight, G.C. White 2nd, M.D. Schaller, SH2and SH3-mediated interactions between focal adhesion kinase and Src, J Biol Chem. 273 (1998) 577-583.

[26] Y. Lim, I. Han, J. Jeon, H. Park, Y.Y. Bahk, E.S. Oh, Phosphorylation of focal adhesion kinase at tyrosine 861 is crucial for Ras transformation of fibroblasts, J Biol Chem. 279 (2004) 29060-29065.

[27] R.D. Read, J.M. Lionberger, T.E. Smithgall, Oligomerization of the Fes tyrosine kinase: Evidence for a coiled-coil domain in the unique N-terminal region. J Biol Chem. 272 (1997) 18498-18503.

[28] Y. Mochizuki, T. Nakamura, H. Kanetake, S Kanda, Angiopoietin 2 stimulates migration and tube-like structure formation of murine brain capillary endothelial cells through c-Fes and c-Fyn. J. Cell Sci. 115 (2002) 175-183.

[29] S. Kanda, T. Suematsu, Y. Miyata, Y. Mochizuki, K. Nomata, H. Kanetake, Sonic hedgehog induces capillary morphogenesis by endothelial cells through phosphoinositide 3-kinase. J. Biol. Chem. 278 (2003) 8244-8249. 
[30] S. Kanda, Y. Mochizuki, H. Kanetake, Stromal cell-derived factor-1 $\alpha$ induces tube-like structure formation of endothelial cells through phosphoinositide 3-kinase. J. Biol. Chem. 278 (2003) 257-262. 


\section{Figure legends}

Fig. 1. FGF-2-treatment decreases the number of focal adhesions (FA) in parental IBE cells, but not in dominant-negative Fes-expressing cells (KE5-15 cells). Cells were seeded onto fibronectin-coated coverslips and cultured in serum-free medium in the presence or absence of $20 \mathrm{ng} / \mathrm{ml} \mathrm{FGF-2} \mathrm{for} \mathrm{the} \mathrm{indicated} \mathrm{periods.} \mathrm{Cells} \mathrm{were} \mathrm{fixed} \mathrm{and} \mathrm{FAs} \mathrm{were} \mathrm{visualized} \mathrm{by} \mathrm{indirect}$ immunofluorescent staining with an anti-vinculin antibody. Values are expressed as the number of focal adhesions/cell \pm SD for at least 200 cells. *; $P<0.05$. Representative images are shown on the left.

Fig. 2. (A) FGF-2 activates Src in IBE cells, but not in KE5-15 cells. Cells were suspended in serum-free medium and seeded onto fibronectin-coated wells of 6-well plates. Cells were cultured for $2 \mathrm{~h}$ in the presence or absence of $20 \mathrm{ng} / \mathrm{ml}$ of FGF-2 and lysed. Src was immunoprecipitated from $80 \%$ of lysates and in vitro kinase assay was performed. Enolase was used as a substrate. The remaining $20 \%$ of each lysate was to assess Src levels by immunoblotting. Comparable data were obtained from two independent experiments. (B) Cells suspended in serum-free medium were seeded onto fibronectin-coated $10 \mathrm{~cm}$ dishes and cultured for $2 \mathrm{~h}$ in the presence or absence of $20 \mathrm{ng} / \mathrm{ml}$ of FGF-2. Cells were lysed and Src was immunoprecipitated followed by immunoblotting for associated Cas. Blots were stripped and reprobed to reveal Cas tyrosine phosphorylation (pTyr), Src protein levels, and Src autophosphorylation (pY418). Comparable data were obtained from two independent experiments. 
Fig. 3. Kinetic study of the tyrosine phosphorylation of FAK. Cells were suspended in serum-free medium and either kept suspended for 15 min (susp) or seeded onto fibronectin-coated wells of $6 \mathrm{~cm}$ dishes to promote adhesion (ad). Cells were cultured for 30 min or $120 \mathrm{~min}$ in the presence or absence of $20 \mathrm{ng} / \mathrm{ml}$ of FGF-2 and lysed. FAK was immunoprecipitated and probed for phosphotyrosine content by immunoblotting. Blots were stripped and reprobed with FAK antibodies to control for FAK recovery. Relative phosphorylation of FAK (the ratio of an intensity of phospho-FAK/total FAK estimated by quantitative densitometry) was calculated and normalized to the ratios observed with untreated, suspended cells (far left lanes). Similar results were obtained from two independent experiments.

Fig. 4. Downregulation of Fes by siRNA abolishes FGF-2-induced Src activation in FA. (A) Fes siRNA-treatment downregulates endogenous Fes in IBE cells. IBE cells grown in $10 \mathrm{~cm}$ dishes were treated with either control siRNA $(10 \mathrm{nM})$ or a mixture of two siRNAs for mouse Fes (10 nM each) and cultured for $48 \mathrm{~h}$. Cells were harvested, counted, and $80 \%$ of cells were used for the determination of Fes expression and the remaining $20 \%$ of cells were used for the expression of $\beta$-actin or indirect immunofluorescent staining (B and $C$ ). (B) Fes is downregulated in IBE cells treated with Fes siRNA. Focal adhesions were visualized by immunostaining with antibodies to vinculin (arrowheads). (C) Anti-pY418 Src phosphospecific antibody detects FGF-2-activated Src within FA. Mock cells (empty vector-transfected cells) or a stable cell line expressing kinase-inactive Src (denoted KDSrc-2 cells) were seeded onto fibronectin-coated coverslips with or without FGF-2 at $20 \mathrm{ng} / \mathrm{ml}$ and cultured for 2 h. Cells were fixed and double stained with anti-vinculin antibody and 
anti-pY418 Src antibody which recognizes the phosphorylated activation loop of c-Src. (D) Src is activated in FA by FGF-2 in IBE cells treated with control siRNA, but not in cells treated with Fes siRNA. Arrowheads indicate the FA. Active Src was visualized by immunostaining with the anti-pY418 antibody. Similar results were obtained in two independent experiments.

Fig. 5. Downregulation of FAK by siRNA abolishes FGF-2-induced Src activation in FA. (A) Downregulation of FAK by siRNA. IBE cells grown in wells of 6-well plates were treated with either control siRNA (5 nM) or the mixture of two siRNA for FAK (1 nM or $5 \mathrm{nM}$ each). Two days later, cells were examined for the expression of FAK by immunoblotting. (B) Cells from part A were examined by indirect immunofluorescent staining for FA with vinculin antibodies and for active Src with phosphospecific antibodies to Tyr 418 as described in the legend to Fig. 4. Arrowheads indicate the FA. Similar results were obtained from two independent experiments.

Fig. 6. Proposed signaling pathways leading to FGF-2-mediated migration of IBE cells. FAK activation is largely regulated by the adhesion of cells to fibronectin. FGF-2 activates Fes and Fes modulates Src activation by FAK. Active Src associates with tyrosine phosphorylated Cas, which in turn regulates focal adhesion turnover and subsequent migration. 

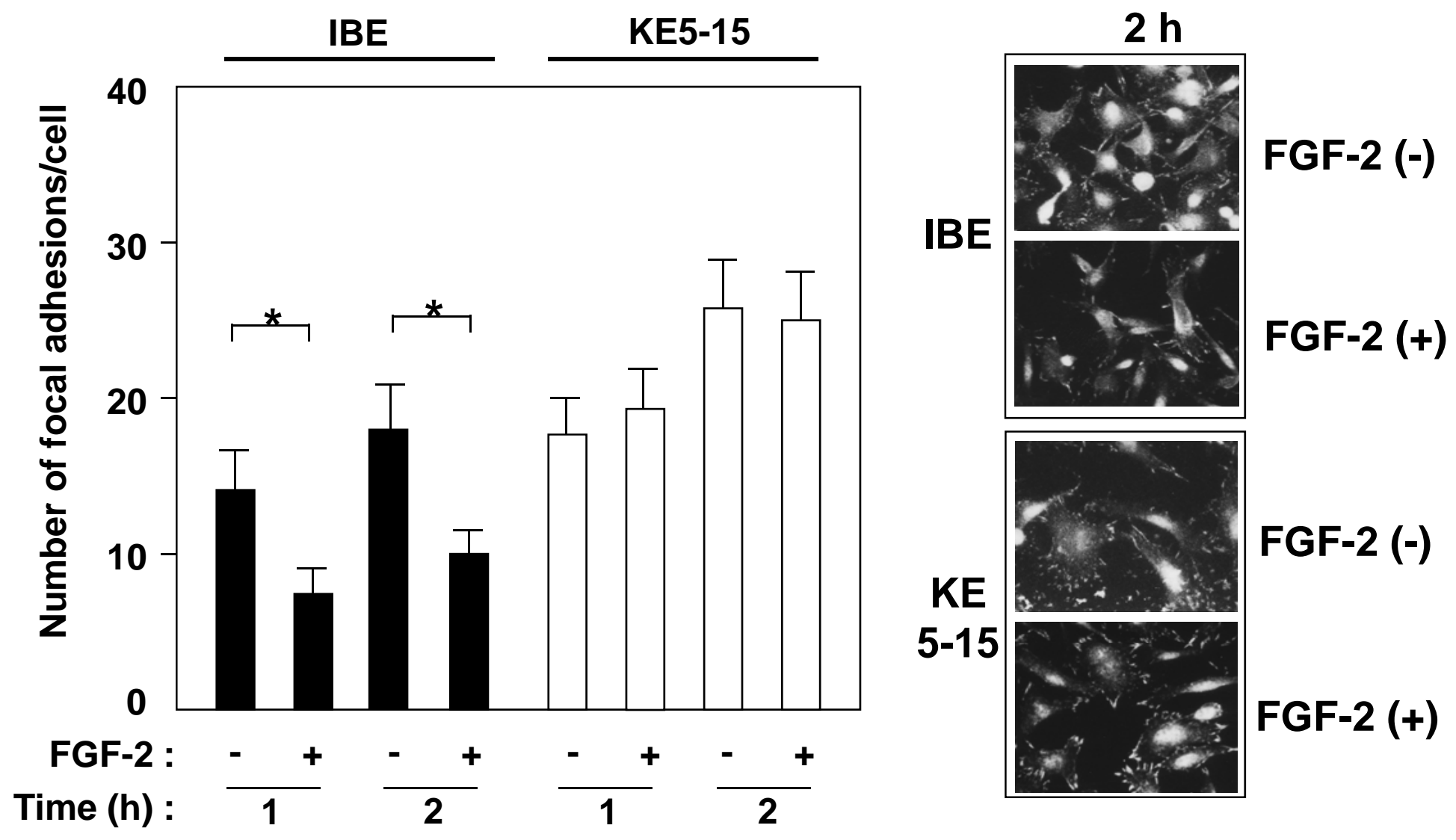

Fig. 1. by S. Kanda et al. 
A

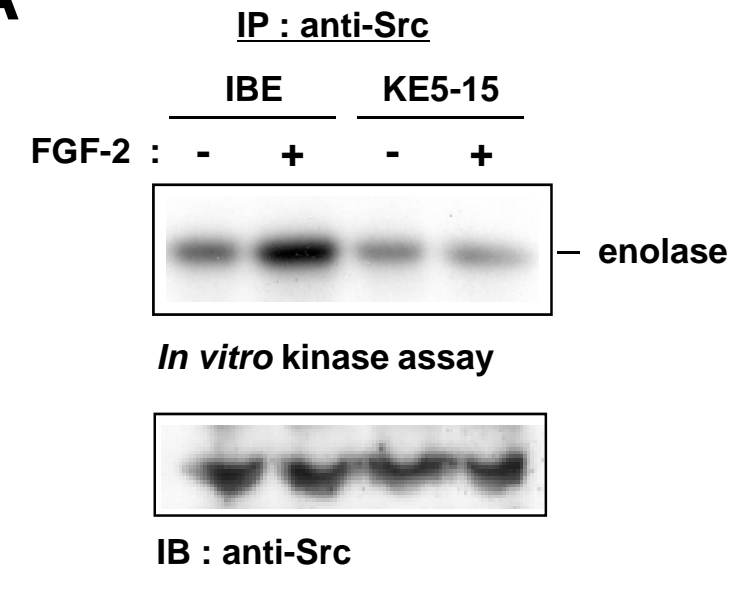

B
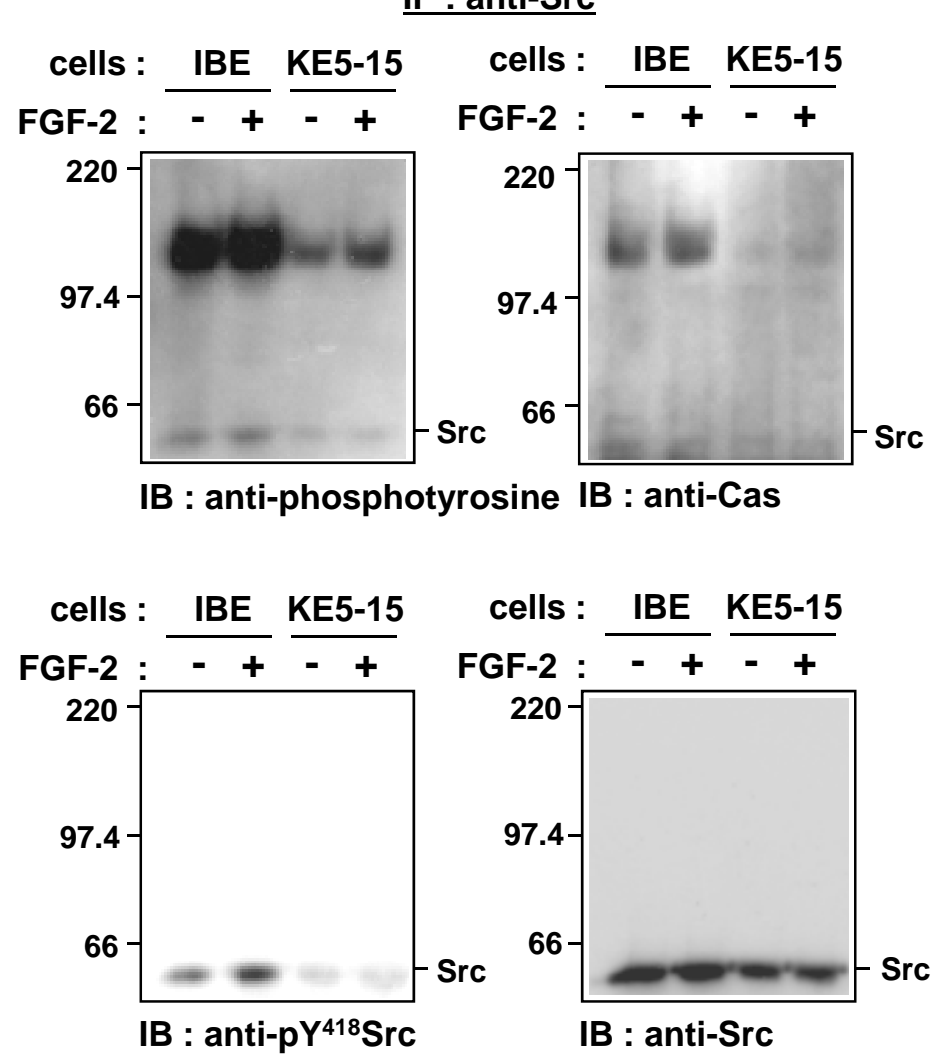

Fig. 2. by S. Kanda et al. 


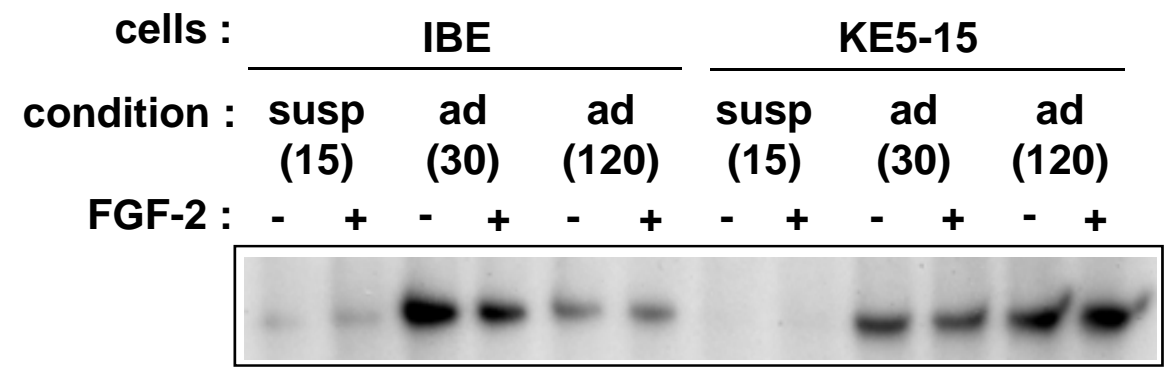

Relative phosphorylation: $1.001 .187 .313 .671 .751 .64 \quad 1.001 .122 .642 .392 .98 \quad 3.03$

$$
\text { IB : anti-pY397 FAK }
$$

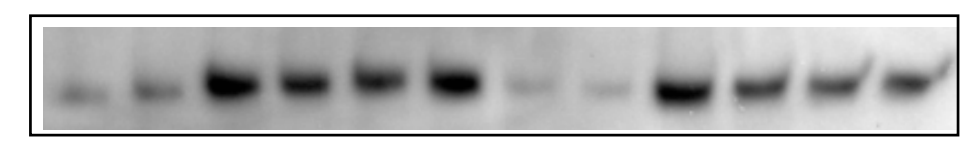

Relative phosphorylation: 1.001 .223 .872 .811 .973 .111 .001 .013 .161 .681 .541 .69 IB : anti-pY861 FAK

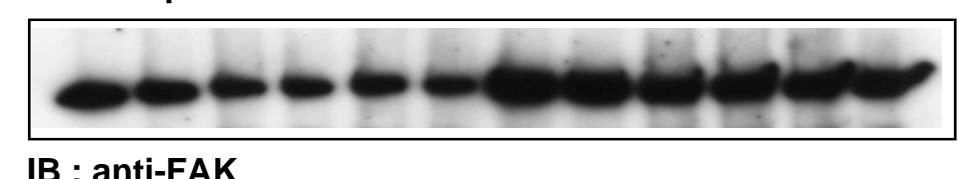

Fig. 3. by S. Kanda et al. 
A cont. siRNA: + -

Fes SiRNA : - +

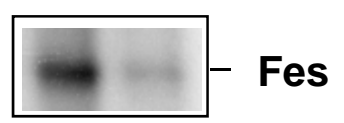

IP and IB : anti-Fes

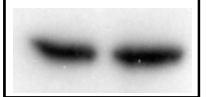

IB : anti- $\beta$-actin

B

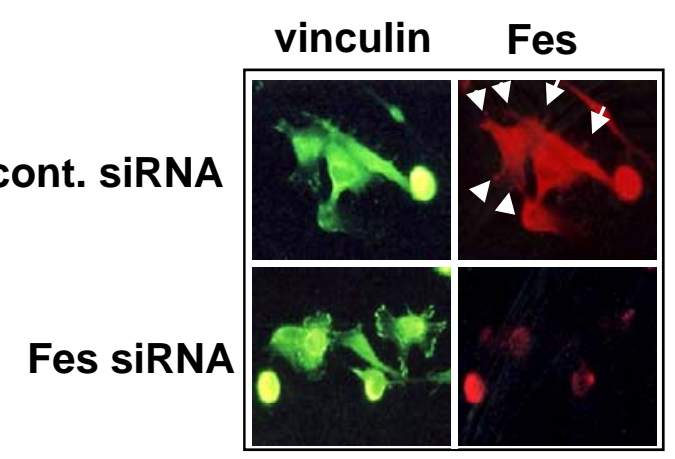

C

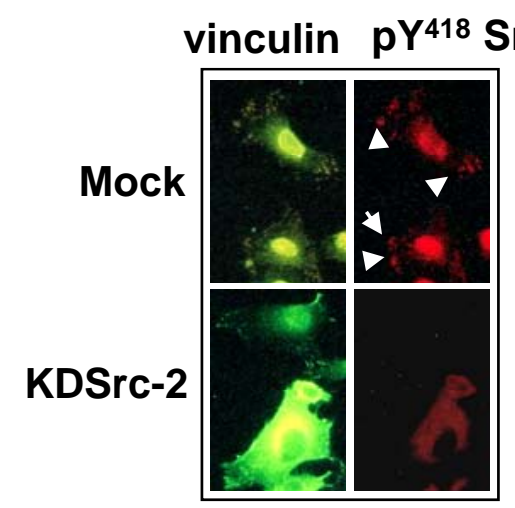

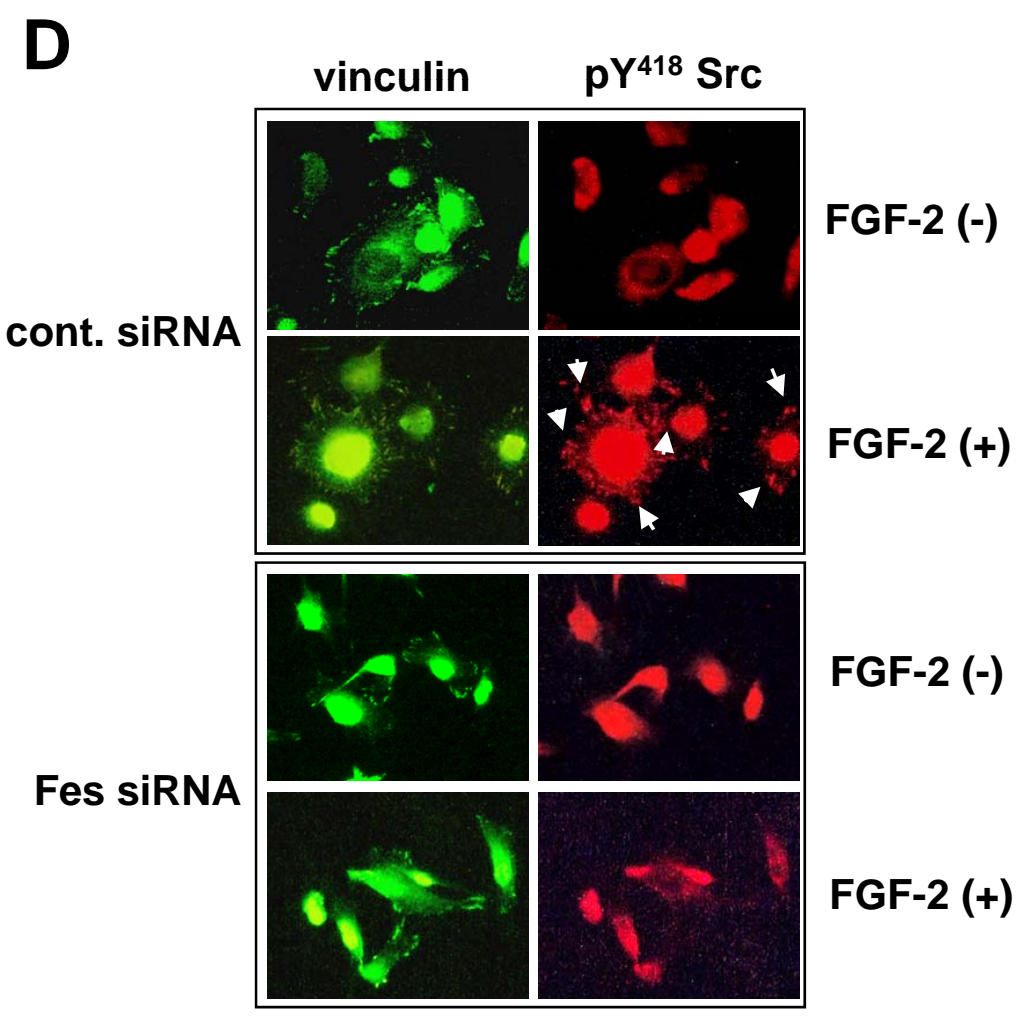

Fig. 4. by S. Kanda et al. 
A

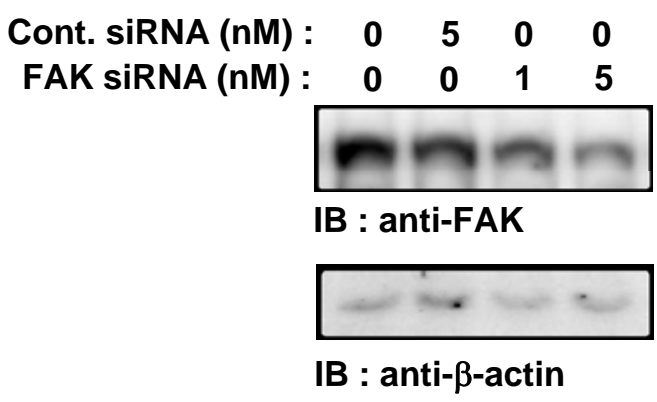

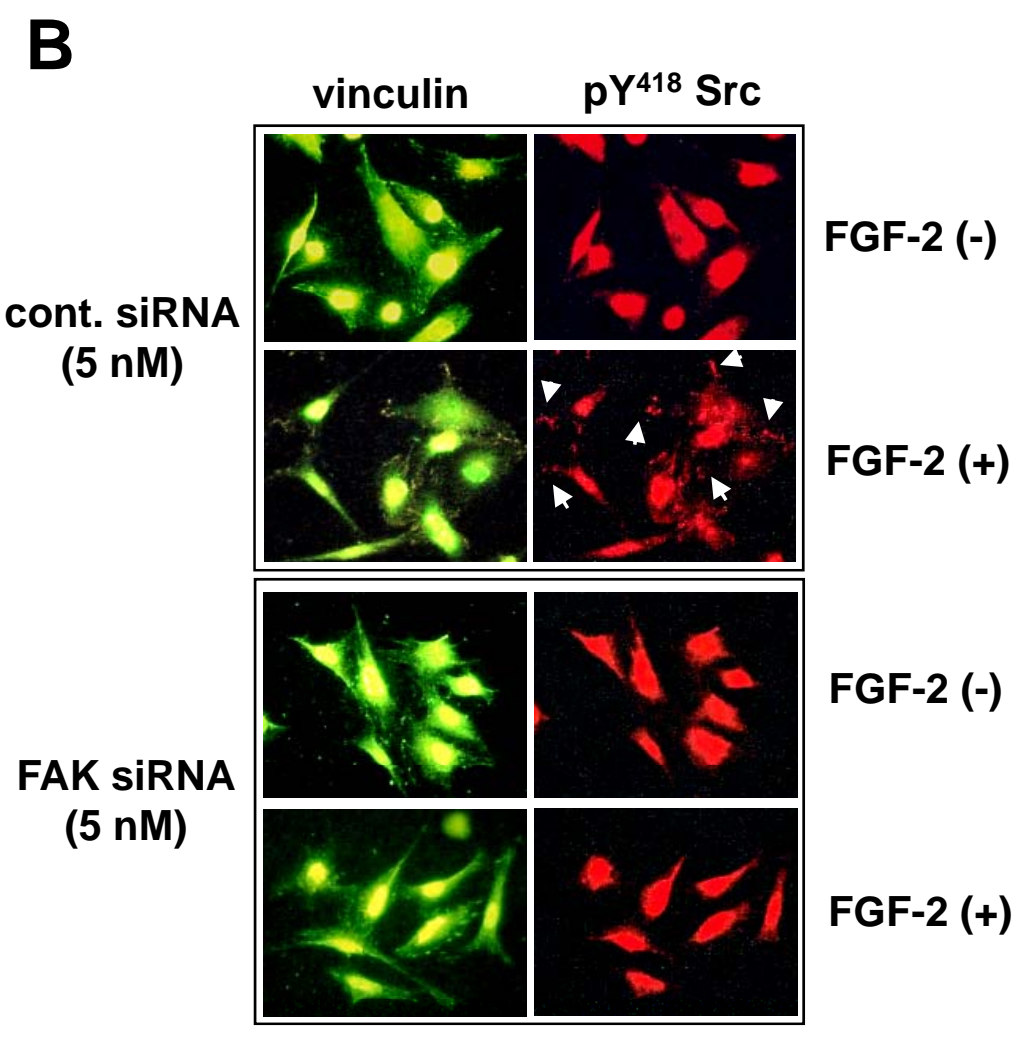

Fig. 5. by S. Kanda et al. 


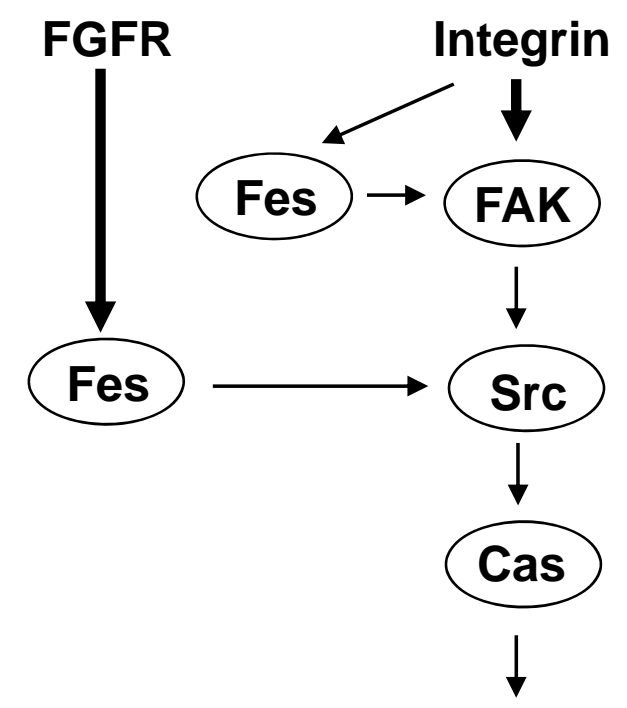

Focal adhesion disassembly

chemotaxis

Fig. 6. S. Kanda et al. 